\title{
Multiorbital antiferromagnetic metal induced by intramolecular self-doping
}

\author{
Rina Takagi, ${ }^{1,2}$ Hiro Gangi, ${ }^{1}$ Kazuya Miyagawa, ${ }^{1}$ Eiji Nishibori,${ }^{3}$ Hidetaka Kasai, ${ }^{3}$ Hitoshi Seo,${ }^{4,5}$ Biao Zhou, ${ }^{6}$ \\ Akiko Kobayashi, ${ }^{6}$ and Kazushi Kanoda ${ }^{1}{ }^{1}$ \\ ${ }^{1}$ Department of Applied Physics, University of Tokyo, Tokyo 113-8656, Japan \\ ${ }^{2}$ Institute of Engineering Innovation, University of Tokyo, Tokyo 113-0032, Japan \\ ${ }^{3}$ Faculty of Pure and Applied Sciences and Tsukuba Research Center for Energy Materials Science (TREMS), \\ University of Tsukuba, Tsukuba 305-8571, Japan \\ ${ }^{4}$ Condensed Matter Theory Laboratory, RIKEN, Saitama 351-0198, Japan \\ ${ }^{5}$ RIKEN Center for Emergent Matter Science (CEMS), Saitama 351-0198, Japan \\ ${ }^{6}$ Department of Chemistry, College of Humanities and Sciences, Nihon University, Tokyo 156-8550, Japan
}

(Received 7 May 2020; accepted 13 July 2020; published 27 August 2020)

\begin{abstract}
The orbital of electrons in a solid affects their mutual interaction, which is a key to emergent phenomena. Therefore, control of the interplay between distinct orbitals makes the behavior of solids fertile. Such a case is substantiated by the aggregation of the molecule, $M(\mathrm{tmdt})_{2}$, which contains $\pi$ orbitals extended over the organic ligand, tmdt, and a level-tunable $d$ orbital centered at the metal ion $M$. Among them, Au(tmdt $)_{2}$ exemplifies the critical $\pi-d$ mixing, which arguably causes an over-100 K antiferromagnetic metal whose nature remains elusive. Here, we track the $\pi-d$ interplay in $\mathrm{Au}(\mathrm{tmdt})_{2}$ with dual orbital-resolved probes, ${ }^{13} \mathrm{C} \mathrm{NMR}$ and synchrotron $\mathrm{x}-$ ray diffraction. We find substantial intramolecular (interorbital) redistribution of electrons on cooling, triggering a commensurate $\pi$ - $d$ antiferromagnetic metal to emerge, which invokes an orbital-selective doped Mott insulator caused by intramolecular self-doping. This demonstrates that vital traffic of electrons between distinct orbitals brings about an unique correlated phase.
\end{abstract}

DOI: 10.1103/PhysRevResearch.2.033321

\section{INTRODUCTION}

The characteristics of orbitals accommodating electrons are the primary determinant of the physical and chemical properties of materials, being of particular significance to strongly interacting electron systems. Materials hosting multiple orbitals that form the bands near the Fermi energy, thanks to their synergistic interplay, harbor a variety of emergent phenomena: multiferroics [1,2], exotic superconductivity with variant pairing symmetries [3] and nematicity [4] in $d$-orbital systems, heavy electron behavior [5,6] and quantum criticality $[7,8]$ in $f$-orbital systems, and so on. These diverse emergent phenomena appear in a material-specific manner depending on what kinds of orbitals are incorporated in what spatial and energetic configuration. In recent years, an unconventional class of molecular materials has opened a distinct channel to the field of multiorbital systems, which we focus on in this work. Conventional molecular conductors are constituted by electron donating and accepting molecular species, either or both of which host partially filled energy bands. This has long been the only way to generate conducting electrons in molecular crystals. The successful synthesis of crystals composed of a single molecular species, $M(\mathrm{tmdt})_{2}$

Published by the American Physical Society under the terms of the Creative Commons Attribution 4.0 International license. Further distribution of this work must maintain attribution to the author(s) and the published article's title, journal citation, and DOI. with a transition-metal ion $M[9,10]$, opened a route to molecule-based electronic conductors in the field of widely studied metal-complex systems such as $(\mathrm{DCNQI})_{2} \mathrm{Cu}$, the tetracyanoplatinates, and halogen-bridged complexes [11-14]. Remarkably, their electronic structure contains several frontier molecular orbitals with discrete characters; some carry the nature of the $d$ orbitals of $M$, but are strongly hybridized with the surrounding sulfur $p$ orbitals, and the others are the $p \pi$ orbitals mostly originated from the ligands, tmdt's $[15,16]$. They are respectively extended in certain portions of the molecule and, by replacing $M$, their linkage is tuned in a systematic way [16-19]. These materials provide unique platforms for multiorbital phenomena in a manner distinct from inorganic crystals.

Planar $M$ (tmdt $)_{2}$ molecules form face-to-face stacking in their crystals [Figs. 1(a) and 1(b)]. Electronic structure calculations show that the $p \pi$ orbitals reside in the vicinity of the Fermi energy $\epsilon_{\mathrm{F}}$, whereas the $d p \sigma$ orbital level varies depending on $M$, as shown in Fig. 1(c) [16-19]. As shown in Fig. 1(c), the $p \pi$ orbital can be viewed as a molecular orbital spatially localized on either of the tmdt ligands. $\mathrm{Ni}(\mathrm{tmdt})_{2}$, which accommodates two electrons in the two $p \pi$ orbitals, is a moderately correlated paramagnetic metal $[9,20]$. On the other hand, in $\mathrm{Cu}(\mathrm{tmdt})_{2}$, the $d p \sigma$ orbital level comes down close to the two $p \pi$ orbitals and all three orbitals accommodate three electrons [19]. There, a peculiar type of multiorbital Mott insulator is realized, with $d p \sigma$ antiferromagnetic (AF) and $p \pi$ spin-gapped Mott subsystems [21-23]. This indicates that the $p \pi$ orbital also bears strong electron-electron correlations. Located in between these two cases is the subject of 

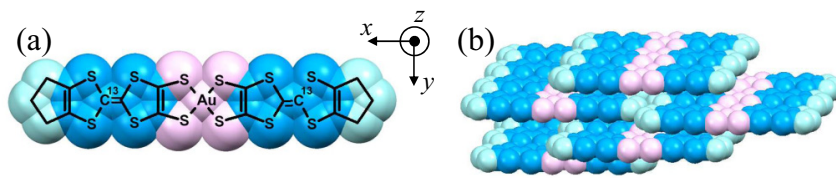

(c)

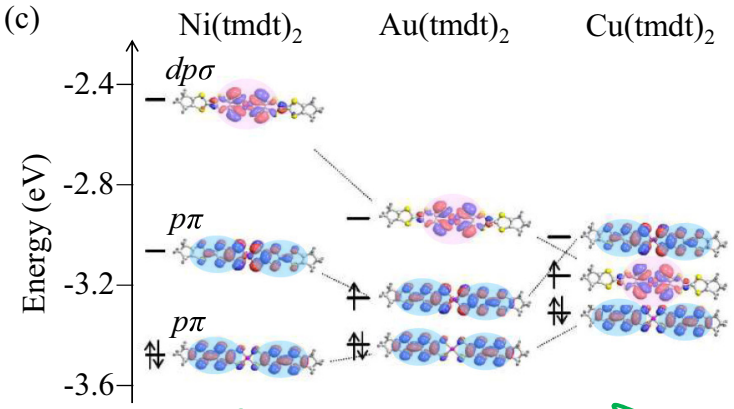

(d)

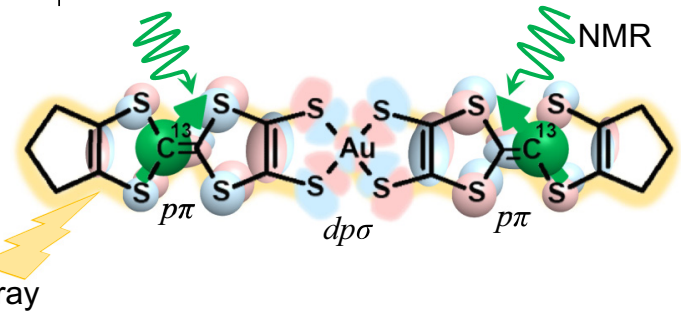

FIG. 1. Structural and orbital properties of $\mathrm{Au}(\mathrm{tmdt})_{2}$. (a) Molecular structure of $\mathrm{Au}(\mathrm{tmdt})_{2}$. The outer parts of the double-bonded carbons at the center of the tmdt ligands are selectively enriched by ${ }^{13} \mathrm{C}$ isotopes. (b) Crystal structure of $\mathrm{Au}$ (tmdt) $)_{2}$. The $p \pi$ orbital is mainly populated in the blue-colored region in the tmdt ligand and the $d p \sigma$ orbital is located in the central $\mathrm{AuS}_{4}$ (pink-colored region). (c) Energy levels of the $p \pi$ and $d p \sigma$ orbitals in $\mathrm{Ni}(\mathrm{tmdt})_{2}, \mathrm{Au}(\mathrm{tmdt})_{2}$, and $\mathrm{Cu}(\mathrm{tmdt})_{2}$ (reproduced from Ref. [19]). (d) Schematics of the present experiments using orbital-resolved probes, ${ }^{13} \mathrm{C}$ NMR and synchrotron $\mathrm{x}$-ray diffraction, to sense the orbital profile of spin density and the molecular structure reflecting charge distribution in the molecule, respectively.

this paper: $\mathrm{Au}(\mathrm{tmdt})_{2}$, in which the $d p \sigma$ orbital level situates above, but near, $\epsilon_{\mathrm{F}}[16-19,24]$.

Surprisingly enough, Au(tmdt $)_{2}$ undergoes an AF transition at an exceptionally high Neel temperature, $T_{\mathrm{N}}$, of $110 \mathrm{~K}$ among molecular materials, while maintaining a metallic state down to the lowest temperature [25-29]. Indeed, the nature of the electronic state is still elusive. At the initial stage a possible spin-density-wave (SDW) formation has been suggested based on the good nesting of the $p \pi$-orbital Fermi surfaces $[17,24]$; however, ${ }^{1} \mathrm{H}$ NMR analysis assuming exclusively the contributions from the $p \pi$ orbital lead to an AF moment of $0.7-1.2 \mu_{\mathrm{B}} / \mathrm{tmdt}$, an unnaturally large value for a SDW [28]. Another scenario would be the strong correlation effect on these $p \pi$ orbitals consistent with the large moments, but in this case it should result in a Mott-insulating state, inconsistent with the transport measurements. An important clue was given by a powder $\mathrm{x}$-ray diffraction measurement finding a gradual structural variation on cooling [29], which affected the energy profile of the orbitals.

Here, we investigate the multiorbital system $\mathrm{Au}(\mathrm{tmdt})_{2}$ through orbital-selective NMR spectroscopy combined with fine-structure-resolvable synchrotron x-ray diffractometry [Fig. 1(d)], which reveals the temperature dependence of spin density in each orbital and unprecedented change of the molecular structure reflecting charge redistribution in the molecule, respectively. The combined results indicate that a $p \pi$-to- $d p \sigma$ electron transfer (interorbital self-doping) is anomalously accelerated upon cooling and results in a commensurate $p \pi-d p \sigma$ hybridized AF order.

\section{EXPERIMENTAL METHODS}

Selectively ${ }^{13} \mathrm{C}$-enriched $\mathrm{Au}(\mathrm{tmdt})_{2}$ was synthesized in a way similar to that for normal $\mathrm{Au}(\mathrm{tmdt})_{2}$, which was reported previously [25]. The ${ }^{13} \mathrm{C}$-enriched tmdt ligands were synthesized from ${ }^{13} \mathrm{CS}_{2}$ according to the literature [30,31].

${ }^{13} \mathrm{C}$ NMR measurements were performed for the fine polycrystals of ${ }^{13} \mathrm{C}$-enriched $\mathrm{Au}(\mathrm{tmdt})_{2}$, where the outer parts of the double-bonded carbons in the center of tmdt were selectively enriched by ${ }^{13} \mathrm{C}$ isotopes as shown in Figs. 1(a) and 1(d). We obtained the NMR spectra through the Fourier transformation of the spin-echo signals and the nuclear spinlattice relaxation curves from the recovery of the echo intensity following saturation comb pulses. Below $T_{\mathrm{N}}$, the width of the spectra was too large to capture the entire spectra in a measurement with the frequency and magnetic field fixed; thus, we constructed the spectra by varying the external magnetic field at a fixed frequency of $139.040 \mathrm{MHz}$.

Single-crystal synchrotron $\mathrm{x}$-ray diffraction experiments were performed at the BL02B1 beamline in SPring-8, Japan [32]. A plate-shaped single crystal of $\mathrm{Au}(\mathrm{tmdt})_{2}$ with dimensions of approximately $15 \mu \mathrm{m}$ was used. We also acquired the diffraction data of the isostructural compound, $\mathrm{Ni}$ (tmdt $)_{2}$, as a reference. Wavelengths of incident x-ray were $0.3865 \AA$ for $\mathrm{Au}(\mathrm{tmdt})_{2}$ and $0.4131 \AA$ for $\mathrm{Ni}(\mathrm{tmdt})_{2}$. The temperature of samples was controlled by a He gas-flow low-temperature device. Integration for Bragg reflections with a resolution of $d>0.5-0.6 \AA$ was carried out using the software Rigaku RAPID AUTO. The structural refinements were performed using SHELX-2013 [33].

\section{RESULTS}

\section{A. Shift analysis}

Figure 2(a) shows the ${ }^{13} \mathrm{C}$ NMR spectra of $\mathrm{Au}(\mathrm{tmdt})_{2}$. At high temperatures above $130 \mathrm{~K}$, the spectral shape is anisotropic and well fitted by the powder average of uniaxially anisotropic Knight shift with the isotropic and anisotropic values, $K_{\text {iso }}$ and $K_{\text {aniso }}$, respectively [Fig. 2(a) and Appendix A]. The uniaxial nature is characteristic of the $p \pi$ orbital, whose spin generates a uniaxial dipole field at the ${ }^{13} \mathrm{C}$ sites through the on-site $p_{z}$ orbital. $K_{\text {iso }}$ potentially has an ambiguity in the chemical-shift determination, whereas $K_{\text {aniso }}$ is free from this problem; so, our analysis focuses on $K_{\text {aniso }}$. Using the known spin susceptibility per tmdt, $\chi / 2$ [26], and the $p \pi$ anisotropic hyperfine coupling constant, $B_{\pi}=3.6 \mathrm{kOe} /\left(\mu_{\mathrm{B}} \mathrm{tmdt}\right)$ [20], we also calculated $B_{\pi} \chi / 2$, which would give $K_{\text {aniso }}$ if the paramagnetic spins were solely on the $p \pi$ orbitals, and plotted them along with the $K_{\text {aniso }}$ values in Fig. 2(b). We note that the $d p \sigma$ anisotropic hyperfine coupling constant, $B_{d}=0.02-$ $0.03 \mathrm{kOe} /\left(\mu_{\mathrm{B}} d p \sigma\right)$ [23], is negligibly small; therefore, $K_{\text {aniso }}$ selectively probes the $p \pi$ spins. $K_{\text {aniso }}$ coincides with $B_{\pi} \chi / 2$ at high temperatures; however, it rapidly deviates downward 

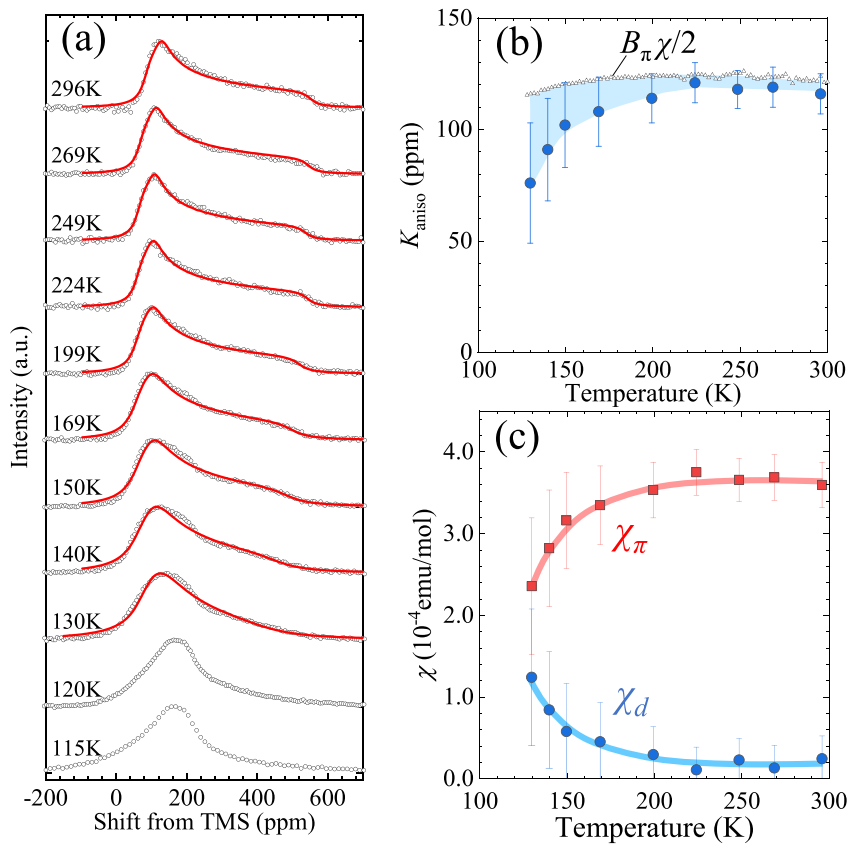

FIG. 2. ${ }^{13} \mathrm{C}$ nuclear magnetic resonance spectra and their analysis for $\mathrm{Au}(\mathrm{tmdt})_{2}$. (a) Temperature variation of the ${ }^{13} \mathrm{C}$ NMR spectra for $\mathrm{Au}(\mathrm{tmdt})_{2}$. The red lines are fits to the spectra [see the text and Appendix A]. (b) Temperature dependence of the anisotropic part of the ${ }^{13} \mathrm{C}$ Knight shift deduced from the spectral fitting and the calculated values of $B_{\pi} \chi / 2$ (open triangle). (c) Spin susceptibilities of the $p \pi$ orbital $\left(\chi_{\pi}\right)$ and the $d p \sigma$ orbital $\left(\chi_{d}\right)$, which are deduced by the analysis of the Knight shift. The error bars in panels (b) and (c) are defined by the inhomogeneous width obtained from the spectral fitting. The red and blue lines in panel (c) are guides to the eye.

from $B_{\pi} \chi / 2$ upon cooling, clearly indicating an accelerated increase in the $d p \sigma$ spin contribution to $\chi$. We can estimate the $d p \sigma$ spin susceptibility $\chi_{d}$ [Fig. 2(c)] by the deviation $\chi-\chi_{\pi}$, where the $p \pi$ contribution is evaluated as $\chi_{\pi}=K_{\text {aniso }} / B_{\pi}$. $\chi_{d}$ is small at high temperatures but progressively increases upon approaching $T_{\mathrm{N}}$ while $\chi_{\pi}$ decreases, demonstrating a gradual electron transfer from the $p \pi$ bands to the $d p \sigma$ band very likely because the two energy levels are getting closer. As temperature is decreased below $130 \mathrm{~K}$, the spectral shape changes [Fig. 2(a)] due to the short-range spin correlation developing toward $T_{\mathrm{N}}$. The analysis of the low-temperature spectra is described later.

\section{B. Relaxation rate}

The ${ }^{13} \mathrm{C}$ nuclear spin-lattice relaxation rate divided by temperature, $\left(T_{1} T\right)^{-1}$, probes the dynamic spin susceptibility. The recovery curve of nuclear magnetization for powdered samples with anisotropic hyperfine coupling as in the present study does not follow a single exponential function of time. Here, we defined the relaxation time $T_{1}$ from the initial slope of the relaxation curve, the inverse of which yields the volume average of different $1 / T_{1}$ 's in randomly oriented microcrystals against the direction of the applied field. As shown in Fig. 3(a), $\left(T_{1} T\right)^{-1}$ is nearly constant between 200 and $300 \mathrm{~K}$; however, it gradually increases with lowering temperature and

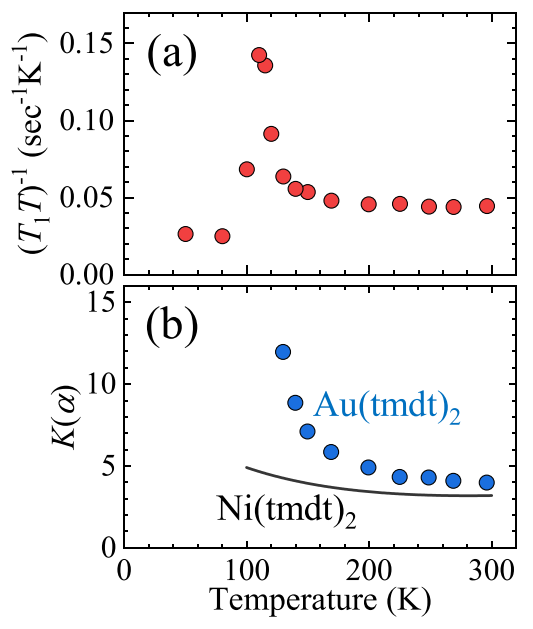

FIG. 3. Spin correlations of $\mathrm{Au}(\mathrm{tmdt})_{2}$. (a) Temperature dependence of the ${ }^{13} \mathrm{C}$ nuclear spin-lattice relaxation rate divided by temperature, $\left(T_{1} T\right)^{-1}$, measured at a magnetic field of $8 \mathrm{~T}$. (b) Temperature dependence of the ${ }^{13} \mathrm{C}$ NMR Korringa ratio $K(\alpha)$. The black line shows the $K(\alpha)$ values for the $p \pi$-band paramagnetic metal

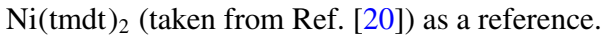

forms a peak around $110 \mathrm{~K}$, reflecting the critical slowing down of the AF fluctuations. The spin fluctuation in a paramagnetic metal is characterized by the so-called Korringa ratio $K(\alpha)$, which is the relaxation enhancement factor in the Korringa relation. For the powder of a crystal with uniaxial hyperfine coupling tensors here, $K(\alpha)$ is given as [34]

$$
\left(T_{1} T\right)^{-1}=K(\alpha) \frac{4 \pi k_{\mathrm{B}}}{\hbar}\left(\frac{\gamma_{\mathrm{e}}}{\gamma_{\mathrm{n}}}\right)^{2}\left(K_{\text {iso }}^{2}+2 K_{\text {aniso }}^{2}\right) .
$$

Using the values of $K_{\text {iso }}$ and $K_{\text {aniso }}$ evaluated above, $K(\alpha)$ is obtained for $T>T_{\mathrm{N}}$ as shown in Fig. 3(b). In weakly correlated metals, the value of $K(\alpha)$ is around unity and is temperature independent, whereas it appreciably deviates from unity in strongly correlated metals [35,36]. For 200 to $300 \mathrm{~K}, K(\alpha)$ takes $\sim 4$, a value close to that of the $p \pi$-band metal Ni(tmdt) ${ }_{2}$ [20] [Fig. 3(b)]. A divergent enhancement of $K(\alpha)$ upon approaching $T_{\mathrm{N}}(=110-120 \mathrm{~K})$ indicates progressive AF fluctuations, a manifestation of strong electron correlation in $\mathrm{Au}(\mathrm{tmdt})_{2}$.

\section{Synchrotron $x$-ray diffraction}

To track the temperature variation of the electron occupations in the $p \pi$ and $d p \sigma$ orbitals, it is useful to examine the intramolecular structure, which is sensitive to the charge distribution profile within the molecule. The tmdt ligand is a tetrathiafulvalene (TTF) derivative, in which the bond lengths of $\mathrm{C}=\mathrm{C}$ and $\mathrm{C}-\mathrm{S}$ are most susceptible to the electron density [37,38]; when it is reduced, the bond length of the bonding (antibonding) orbital on $\mathrm{C}=\mathrm{C}(\mathrm{C}-\mathrm{S}), d_{\mathrm{C}=\mathrm{C}}\left(d_{\mathrm{C}-\mathrm{S}}\right)$, is elongated (shortened). The amount of electron transfer is empirically evaluated from the ratio $\gamma=d_{\mathrm{C}=\mathrm{C}} / d_{\mathrm{C}-\mathrm{S}}$ and the difference $\delta=d_{\mathrm{C}-\mathrm{S}}-d_{\mathrm{C}=\mathrm{C}}[37,38]$. According to the molecular-orbital calculations for $M(\mathrm{tmdt})_{2}$, the profile of the $p \pi$ orbital and its bonding character are similar to those 


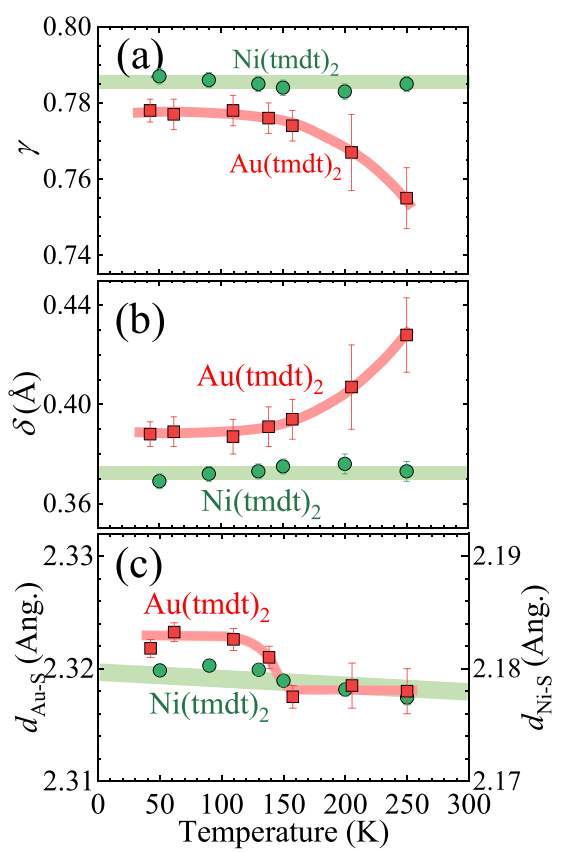

FIG. 4. Unusual structure variation in $\mathrm{Au}(\mathrm{tmdt})_{2}$. Temperature dependencies of the ratio between the $\mathrm{C}=\mathrm{C}$ and $\mathrm{C}-\mathrm{S}$ bond lengths, $\gamma=d_{\mathrm{C}=\mathrm{C}} / d_{\mathrm{C}-\mathrm{S}}$ (a), their difference, $\delta=d_{\mathrm{C}-\mathrm{S}}-d_{\mathrm{C}=\mathrm{C}}(\mathrm{b})$, and the $M$-S bond length $d_{M-\mathrm{S}}$ (c). The red squares are for $\mathrm{Au}(\mathrm{tmdt})_{2}$ and the green circles are for $\mathrm{Ni}(\mathrm{tmdt})_{2}$. These bond lengths are sensitive to the profile of charge distribution in the molecule (see text).

of other TTF derivatives; thus, we use $\gamma$ and $\delta$ as indices characterizing the electron occupation in tmdt.

Figures 4(a) and 4(b) show the temperature dependencies of $\gamma$ and $\delta$ for $\mathrm{Au}(\mathrm{tmdt})_{2}$ and $\mathrm{Ni}(\mathrm{tmdt})_{2}$ deduced from the synchrotron x-ray diffraction data. Here, we estimated $d_{\mathrm{C}=\mathrm{C}}$ and $d_{\mathrm{C}-\mathrm{S}}$ from the average of $\mathrm{C}=\mathrm{C}$ and $\mathrm{C}-\mathrm{S}$ bond lengths in the tmdt ligands, respectively. For $\mathrm{Au}(\mathrm{tmdt})_{2}, \gamma$ and $\delta$ show clear increases and decreases upon cooling, respectively, both reaching constant values when $T_{\mathrm{N}}$ is approached. These results suggest that electron occupation in tmdt decreases, meaning an electron transfer from the $p \pi$ orbital to the $d p \sigma$ orbital. To check other possible contributions for the temperature variation of the bond lengths, such as thermal contraction, we did the same experiment and analysis for $\mathrm{Ni}(\mathrm{tmdt})_{2}$. It is evident in Figs. 4(a) and 4(b) that changes in $\gamma$ and $\delta$ for $\mathrm{Au}(\mathrm{tmdt})_{2}$ are 1 order of magnitude larger than those in $\mathrm{Ni}(\mathrm{tmdt})_{2}$; this phenomenon is specific to $\mathrm{Au}(\mathrm{tmdt})_{2}$. To confirm the intramolecular electron transfer, we examined the temperature dependence of the $M-\mathrm{S}$ bond length. It is expected to elongate when the electrons accumulate more in the $d p \sigma$ orbital, which is roughly regarded as an antibonding combination of the metal site $d_{x y}$ orbital and the surrounding S $3 p$ orbitals $[17,18]$. Figure 4(c) shows that, upon cooling, this is actually the case. The amount of intramolecular electron transfer upon cooling is roughly estimated as 0.36$0.38 e / \mathrm{tmdt}(0.72-0.76 e / d p \sigma)$ from $\gamma$ and $\delta$ (Appendix C), an extremely large value consistent with the unusual behavior of the NMR shift. We note that such an intramolecular electron transfer is associated with a valence change of the Au ion and
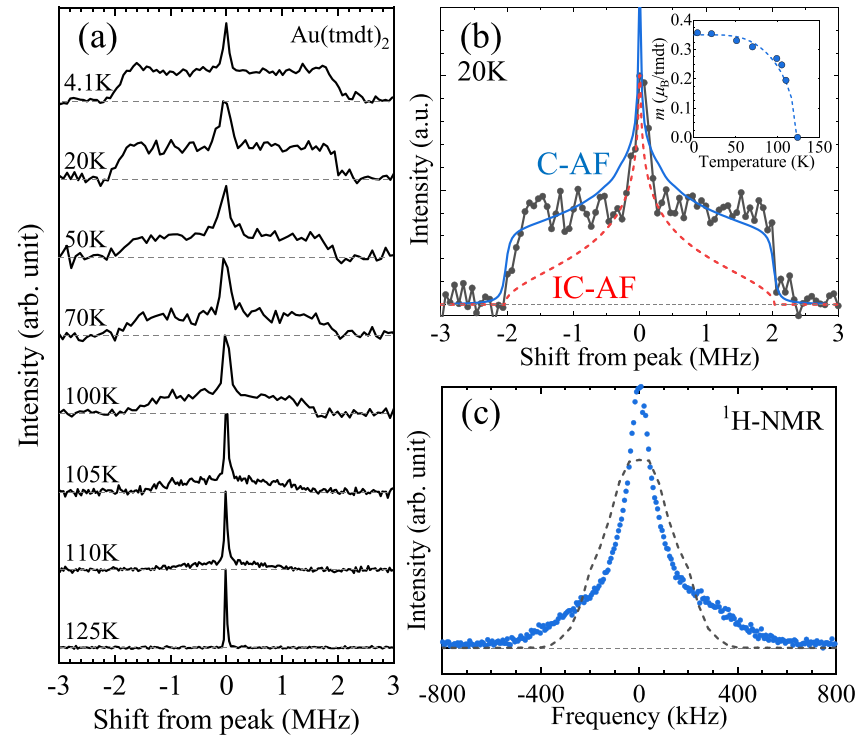

FIG. 5. ${ }^{13} \mathrm{C}$ and ${ }^{1} \mathrm{H}$ NMR spectra in the antiferromagnetic state. (a) Temperature dependence of the ${ }^{13} \mathrm{C}$ NMR spectra of $\mathrm{Au}(\mathrm{tmdt})_{2}$, constructed by measuring the echo intensity as a function of magnetic field under a fixed resonance frequency. (b) ${ }^{13} \mathrm{C}$ NMR spectra of $\mathrm{Au}(\mathrm{tmdt})_{2}$ at $20 \mathrm{~K}$ and the simulated spectra for a powdered sample with the commensurate $\mathrm{AF}$ state $(\mathrm{C}-\mathrm{AF})$ and the incommensurate AF state (IC-AF). Temperature evolution of the order parameter $m$ is shown in the inset, where the dashed curve corresponds to the Brillouin function for $S=1 / 2$. (c) The simulated ${ }^{1} \mathrm{H}$ NMR spectrum for an $\mathrm{AF}$ order of the moment of $0.35 \mu_{\mathrm{B}}$ per tmdt ligand with a wave vector, $q=(\pi, 0,0)$ (black dashed line), and the experimental ${ }^{1} \mathrm{H}$ NMR spectrum at $2.1 \mathrm{~K}$ (blue dots) taken from Ref. [28].

thus is possibly related to the multiplicity of the Au oxidation states, i.e., $\mathrm{Au}^{1+}$ and $\mathrm{Au}^{3+}$.

\section{AF order spectra}

The behavior of the $p \pi$ and $d p \sigma$ spin susceptibilities points to the possibility that the AF ordered moments have a dual nature. Below $T_{\mathrm{N}}$, the NMR spectra steeply become broadened [Fig. 5(a)] and, at the lowest temperature, spread symmetrically with clear edges at $\pm 2.1 \mathrm{MHz}$, superposed by a sharp central peak. As mentioned above, the hyperfine coupling constant of the ${ }^{13} \mathrm{C}$ nucleus with the $p \pi$ spin is 100 -fold larger than that with the $d p \sigma$ spin $[20,23]$; thus the spectral broadening is attributed to the $p \pi$ spins. To evaluate the size of the ordered moment, we simulated ${ }^{13} \mathrm{C}$ NMR powder spectra, assuming that the AF moments are flopped to arbitrary directions in the plane normal to the applied field (see Appendix A). The simulation reproduces the precipitous edges and a central peak as shown in Fig. 5(b), with a moment of $0.35 \mu_{\mathrm{B}}$ per tmdt. (If the spectral broadening were solely due to the $d p \sigma$ spins, a totally unphysical value of 10-24 $\mu_{\mathrm{B}}$ would result.) An incommensurate AF order as in an SDW state is ruled out, since it would show no edges, as shown in Fig. 5(b). The temperature evolution of the moment is shown in the inset, following the mean-field behavior. In the lowtemperature limit, the moment is much larger than $0.08 \mu_{\mathrm{B}}$ in a SDW compound, (TMTSF) ${ }_{2} \mathrm{PF}_{6}$ [39], and comparable 
to $0.45 \mu_{\mathrm{B}}$ in a Mott insulator, $\kappa-(\mathrm{ET})_{2} \mathrm{Cu}\left[\mathrm{N}(\mathrm{CN})_{2}\right] \mathrm{Cl}$ [40], suggesting that electron correlation drives the $\mathrm{AF}$ order in $\mathrm{Au}(\mathrm{tmdt})_{2}$.

The AF moment previously deduced from the ${ }^{1} \mathrm{H}$ NMR spectra assuming solely the $p \pi$ spins yields $0.7-1.2 \mu_{\mathrm{B}} / \mathrm{tmdt}$ [28], a twofold larger value than the present result. This discrepancy implies an additional contribution from the $d p \sigma$ spins to the ${ }^{1} \mathrm{H}$ NMR spectra missed in the previous analysis. The ${ }^{1} \mathrm{H}$ NMR spectra are shaped by anisotropic dipole fields from the $p \pi$ and $d p \sigma$ spins flopped perpendicular to the applied field, where the isotropic hyperfine fields have no impact on the spectra. Using the molecular-orbital calculations of spin density profile, the dipole fields at $\operatorname{six}{ }^{1} \mathrm{H}$ sites in tmdt are calculated as ${ }^{\mathrm{H}} B_{\pi}=0.05-0.18 \mathrm{kOe} /\left(\mu_{\mathrm{B}}\right.$ tmdt- $\left.p \pi\right)$ and ${ }^{\mathrm{H}} B_{d}=0.11-0.19 \mathrm{Oe} /\left(\mu_{\mathrm{B}} d p \sigma\right)$, meaning that ${ }^{1} \mathrm{H}$ equally senses the $p \pi$ and $d p \sigma$ spins; note that the nearest $d p \sigma$ spin is on the adjacent molecule. The simulation of the ${ }^{1} \mathrm{H}$ spectra is, however, much more complicated than the ${ }^{13} \mathrm{C}$ case, because the dipole field at ${ }^{1} \mathrm{H}$ also contains large components from further neighbor $p \pi$ and $d p \sigma$ orbitals, differently from site to site among the six ${ }^{1} \mathrm{H}$ 's, so that the summed ${ }^{1} \mathrm{H}$ spectral shape heavily depends on the AF wave vector. Indeed, our simulations of ${ }^{1} \mathrm{H}$ powder spectra for 14 possible AF configurations of the $p \pi$ spins alone are very sensitive to the AF configuration and importantly none of them reproduces the observed line shape; neither do the simulations for the $d p \sigma$ spins [see Appendix B]. Figure 5(c) illustratively shows a simulation for a theoretically proposed configuration [16-18,24] for 0.35 $\mu_{\mathrm{B}} /$ tmdt together with the observed spectra at $2.1 \mathrm{~K}$; the discrepancy implies the subtractive (additive) contributions of the $d p \sigma$ moments to the central (outer) parts of the spectrum. The second moment, a measure of the squared linewidth, is $(182 \mathrm{kHz})^{2}$ and $(131 \mathrm{kHz})^{2}$ for the observed and simulated spectra, respectively. We can roughly ascribe the extra width, $126\left[=\left(182^{2}-131^{2}\right)^{1 / 2}\right] \mathrm{kHz}$, to the $d p \sigma$ moment, which is estimated as $0.21 \mu_{\mathrm{B}}$ referring to the separately examined linewidth- $d p \sigma$ moment proportionality, $600 \mathrm{kHz} /\left(\mu_{\mathrm{B}} d p \sigma\right)$. The other spin configurations provide similar estimates, so we can conclude that the $d p \sigma$ spins also carry sizable AF moments.

\section{DISCUSSION}

The present results provide insight into the electronic state of $\mathrm{Au}(\mathrm{tmdt})_{2}$ and a promising nature of the enigmatic AF phase transition. According to the electronic structure calculations based on the room-temperature crystal structure [16-19,24], the $d p \sigma$ orbital level resides above $\epsilon_{\mathrm{F}}$, of the order of $0.1 \mathrm{eV}$, so that the two tmdt $p \pi$ orbitals in a molecule accommodate three electrons, forming a 3/4-filled $p \pi$ band [Fig. 6(a)]. Indeed, this situation is evidenced by the spin susceptibility that comes exclusively from the $p \pi$ spins at high temperatures. Upon cooling, our results show that the spin susceptibility gradually transfers to the $d p \sigma$ orbital, indicating an increasing hybridization of the two kinds of orbitals at $\epsilon_{\mathrm{F}}$; this is indeed consistent with the lowering of the $d p \sigma-$ orbital level estimated based on the low-temperature structure $[16,29]$. Upon approaching $T_{\mathrm{N}}$, this tendency is accelerated by a rapid evolution of the hybridization, and $\chi_{\pi}$ and $\chi_{d}$ become comparable in magnitude near $T_{\mathrm{N}}$, indicating substantial

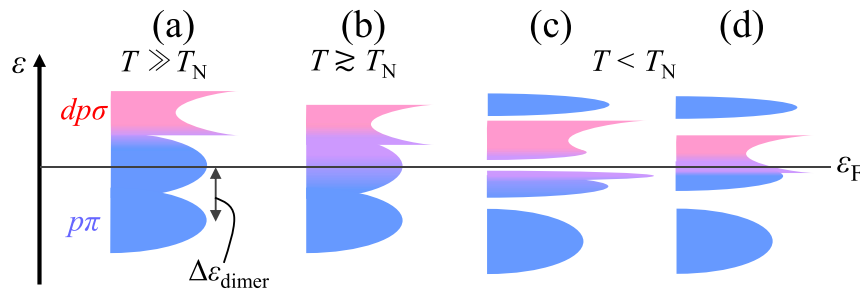

FIG. 6. Schematic electronic density of states for the temperature evolution of the $p \pi-d p \sigma$ band mixing in $\mathrm{Au}(\mathrm{tmdt})_{2}$. (a) For $T \gg$ $T_{\mathrm{N}}$, the one-dimensional $d p \sigma$ band resides well above the Fermi energy $\epsilon_{\mathrm{F}}$, and the two two-dimensional $p \pi$ bands accommodate all electrons, forming a $3 / 4$-filled $p \pi$ band. The dimerization in degenerated $p \pi$ orbitals is represented by the intermolecular dimerization splitting $\Delta \epsilon_{\text {dimer }}$, making the antibonding-like and bonding-like $p \pi$ bands. (b) Upon cooling, the $d p \sigma$ band comes down in energy, resulting in increasing the hybridization of the $d p \sigma$ band to the $p \pi$ band at $\epsilon_{\mathrm{F}}$ for $T \geqslant T_{\mathrm{N}}$. (c), (d) As the $d p \sigma$ band further comes down in energy, upon further cooling it gets hybridized to the upper-located antibonding $p \pi$ band, which possibly leads to a band splitting due to enhanced correlation. Two conceivable cases in which the bands have a gap at $\epsilon_{\mathrm{F}}(\mathrm{c})$ or not (d) are depicted.

hybridization (and overlapping of the $p \pi$ and $d p \sigma$ bands) [Fig. 6(b)]. Below $T_{\mathrm{N}}$, large magnetic moments appear in both $p \pi$ and $d p \sigma$ orbitals and order commensurately, while keeping the metallic conduction. On the basis of these results, we discuss the electronic state of $\mathrm{Au}(\mathrm{tmdt})_{2}$ in the following.

It has been shown that one of the intermolecular ligand $p \pi-p \pi$ transfer integral in the unit cell is larger than the others, including the intramolecular one, so that the tmdt's connected by this transfer integral can be viewed as dimers. Their bonding and antibonding orbitals basically constitute the $p \pi$ bands with a dimerization splitting, $\Delta \epsilon_{\text {dimer }}$ [16], as shown in Fig. 6(a). Then, the antibonding bands are effectively half-filled and they are susceptible to the so-called dimer-Mott-insulating state, as discussed in many organic conductors [41].

Now, when the $d p \sigma$ band drops in energy, as revealed by the present measurements, it hybridizes to the upper-located antibonding $p \pi$ band. We expect that the system becomes more strongly correlated because the hybridized $d p \sigma$ orbital is further spatially confined than the $p \pi$ orbital and then should have larger on-site Coulomb repulsion. The AF order emerges from the reconstructed bands from the antibonding$p \pi$ and $d p \sigma$ bands. A key to the origin of the AF order is its commensurate nature, which, in conjunction with the large AF moment, suggests the strong correlation effect as the driving force of the AF order. Let us discuss possible scenarios based on the multiband Hubbard model for $M(\text { tmdt })_{2}$; in fact, meanfield analysis shows a commensurate AF metal with moments in both $p \pi$ and $d p \sigma$ orbitals, when the orbital hybridization is large [16]. There, the $p \pi$ orbitals show a dimer-Mott-type AF order with a large moment that can reach about $0.4 \mu_{\mathrm{B}}$. And by the electron transfer from them, the $d p \sigma$ orbitals also host spin ordering. Considering the quantum effect beyond mean-field approximation, which is indispensable for the understanding of strongly correlated systems $[6,7,42]$, the Coulomb interaction should enhance the Mottness of the $d p \sigma$-hybridized $p \pi$ band and lead to a band splitting. This is supported by the fact 
that all bands made of two $p \pi$ orbitals and a $d p \sigma$ orbital are Mott insulating in $\mathrm{Cu}$ (tmdt $)_{2}$ [23]. Two cases are conceivable; the bands have a gap at $\epsilon_{\mathrm{F}}$ [Fig. 6(c)] or not [Fig. 6(d)]. The former, however, is inconsistent with the metallic behavior of $\mathrm{Au}(\mathrm{tmdt})_{2}$, whereas, in the latter case, the lower (so-called Hubbard) $p \pi$ band is hole-doped and achieves metallicity. The latter case can be considered as an orbital-selective doped Mott insulator, which we propose to be realized in the lowtemperature state of $\mathrm{Au}(\mathrm{tmdt})_{2}$. Theoretically, the concept of orbital-selective Mottness has been intensively investigated in multiorbital materials such as iron-based superconductors and heavy-fermion systems [42]. We also note that a doped Mott insulator is predicted to be able to host a commensurate AF order and metallicity simultaneously by a study of the $t-J$ model [43]. Further study by optical spectroscopy is a promising way to experimentally identify the effect of electron correlations, such as the formation of a Mott gap.

\section{SUMMARY}

In the present work, a prominent feature is that the system spontaneously provokes a notable amount of intramolecular electron redistribution, which promotes a vital $\pi-d$ interplay bringing forth an emergent correlated phase. This is unique among $\pi-d$ electron systems, most of which are constituted by molecular species separately hosting $\pi$ and $d$ orbitals with perturbative $\pi$ - $d$ hybridization due to separation of the orbitals in space and energy. An analogous example to the present case is (DCNQI $)_{2} \mathrm{Cu}$, in which one-dimensional chains of DCNQI $\pi$ orbitals are bridged by $d$ orbitals of $\mathrm{Cu}$ ions [11,44]. Physical or chemical pressure causes a substantial charge transfer between the two orbitals, which helps to cooperatively stabilize the Peierls instability in the one-dimensional $\pi$ orbital and a charge order in the $\mathrm{Cu}$ sites $[45,46]$. In the present system, Au(tmdt) ${ }_{2}$, two-dimensional networks of $\pi$ orbitals are connected through one-dimensional chains of $d$ orbitals [Fig. 1(b)] and a Mott phase results. These two contrasting cases demonstrate that a variety of phases are induced by the $\pi-d$ electron redistribution through self-doping between distinct bands, which unveils emergent phases underlying but otherwise hidden in condensed matters.

\section{ACKNOWLEDGMENTS}

The authors thank S. Ishibashi for helpful discussions and K. Sugimoto for experimental support at SPring-8. The synchrotron x-ray experiments were conducted at the BL02B1 beamline at SPring-8 (Proposal No. 2015A1317). This work was partly supported by JSPS Grants-In-Aid for Scientific Research (Grants No. 17K05532, No. 17K05846, No. 18H05225, No. 19H01846, No. 19K21860, and No. 19K03723).

\section{APPENDIX A: SHIFTS FROM SPECTRAL FITTING}

In the paramagnetic state, the shift of the ${ }^{13} \mathrm{C} N M R$ line under a field described in spherical coordinates, $(\theta, \phi)$, is expressed by $\delta(\theta, \phi)=\delta_{x x} \sin ^{2} \theta \cos ^{2} \phi+\delta_{y y} \sin ^{2} \theta \sin ^{2} \phi+$ $\delta_{z z} \cos ^{2} \theta$, where $x$ and $y$ axes are the long and short axes in the tmdt plane and the $z$ axis is normal to the plane as shown in Fig. 1(a) and $\delta_{i i}(i=x, y, z)$ is the $i$-axis principal value of the shift tensor. Then, the spectral shape of the powder pattern convoluted by the Lorentzian function with the inhomogeneous width $\Delta$ is expressed as

$$
F(v)=Z \iint \frac{\Delta}{\left(\delta_{x x} \sin ^{2} \theta \cos ^{2} \phi+\delta_{y y} \sin ^{2} \theta \sin ^{2} \phi+\delta_{z z} \cos ^{2} \theta-v\right)^{2}+\Delta^{2}} \sin \theta d \theta d \phi,
$$

where $Z$ is a normalization factor. The shift of the NMR line consists of the chemical shift and the spin shift; the former depends on the local chemical structure around the nuclear site, whereas the latter is related to the local spin susceptibility. The total shift tensor is described as

$$
\begin{aligned}
\left(\begin{array}{ccc}
\delta_{x x} & 0 & 0 \\
0 & \delta_{y y} & 0 \\
0 & 0 & \delta_{z z}
\end{array}\right)= & \sigma_{\text {iso }}+\left(\begin{array}{ccc}
\sigma_{1} & 0 & 0 \\
0 & \sigma_{2} & 0 \\
0 & 0 & -\sigma_{1}-\sigma_{2}
\end{array}\right)+K_{\text {iso }} \\
& +K_{\text {aniso }}\left(\begin{array}{ccc}
-1 & 0 & 0 \\
0 & -1 & 0 \\
0 & 0 & 2
\end{array}\right)
\end{aligned}
$$

The first and second (third and fourth) terms are the isotropic and anisotropic components of the chemical shift (spin shift). The anisotropic spin shift tensor is assumed to be uniaxial around the $z$ axis due to uniaxial symmetry of the dipolar hyperfine tensor stemming from the carbon-site $2 p_{z}$ orbitals, which constitute the $p \pi$ orbitals on tmdt. The inhomogeneous width $\Delta$ is assumed to have a form of $\Delta=\Delta_{0}+\Gamma \mid K_{\text {iso }}-$ $K_{\text {aniso }}\left(\sin ^{2} \theta \cos ^{2} \phi+\sin ^{2} \theta \sin ^{2} \phi-2 \cos ^{2} \theta\right) \mid$, which consists of the constant term $\Delta_{0}$ and the second term proportional to the Knight shift with prefactor $\Gamma$.

First, we evaluated the chemical shift tensor $\left(\sigma_{\text {iso }}, \sigma_{1}\right.$, and $\sigma_{2}$ ) by fitting the spectrum for $296 \mathrm{~K}$. To reduce the number of fitting parameters, we determined the total isotropic component $\left(=\sigma_{\text {iso }}+K_{\text {iso }}\right)$ from the center of gravity of the spectrum and fixed $K_{\text {aniso }} / K_{\text {iso }}$ to 0.8 , which was previously determined for $\mathrm{Ni}(\mathrm{tmdt})_{2}$ at room temperature. An excellent fit with $\sigma_{\text {iso }}=114, \sigma_{1}=-55$, and $\sigma_{2}=-9$ is seen in Fig. 2(a). Then, fixing the values of $\sigma_{\text {iso }}, \sigma_{1}$, and $\sigma_{2}$ to those, we proceeded to fitting for other temperatures with $K_{\text {iso }}, K_{\text {aniso }}, \Delta$, and $\Gamma$ as fitting parameters.

In the antiferromagnetic state, spectral simulations were performed under the assumption that the magnetic moments are directed in the plane perpendicular to the external magnetic field $\boldsymbol{H}_{0}=H_{0}(\cos \phi \sin \theta, \sin \phi \sin \theta, \cos \theta)$ due to the spin flop. Assuming that the spin direction is arbitrary in the plane, the magnetic moment $s$ is expressed as $\boldsymbol{s}=|\boldsymbol{s}|(\cos \phi \cos \theta \cos t-\sin \phi \sin t, \sin \phi \cos \theta \cos t+$ $\cos \phi \sin t,-\sin \theta \cos t)$, where $t$ represents the direction of the moments in the plane. We employed the hyperfine coupling tensor $\boldsymbol{A}$ at the ${ }^{13} \mathrm{C}$ nuclei with uniaxial anisotropy for the $z$ 
axis [Fig. 1(a)], which is described as

$$
\boldsymbol{A}=a_{\text {iso }}+a_{\text {aniso }}\left(\begin{array}{ccc}
-1 & 0 & 0 \\
0 & -1 & 0 \\
0 & 0 & 2
\end{array}\right) \text {. }
$$

Here, we used the hyperfine coupling constants $\left(a_{\text {iso }}, a_{\text {aniso }}\right)$ $=(4.3,3.6) \mathrm{kOe} /\left(\mu_{\mathrm{B}}\right.$ tmdt $)$ [20]. The $\boldsymbol{H}_{0}$-parallel component of the local field generated by a magnetic moment of $1 \mu_{\mathrm{B}} /$ tmdt, $h(\theta, \phi, t)$, is given by $h=\boldsymbol{H}_{0} \cdot \boldsymbol{A} \cdot \boldsymbol{s} / H_{0}|\boldsymbol{s}|$. To obtain the spectra for the polycrystalline sample, we summed up the spectral contributions from grain to grain, in which $t$ is also averaged uniformly in $0 \leqq t<2 \pi$. Thus, the spectral function is described as

$$
F(v)=\iiint \frac{\Delta}{[v-\gamma h(\theta, \phi, t)]^{2}+\Delta^{2}} \sin \theta d \theta d \phi d t,
$$

where $\gamma$ is the gyromagnetic ratio of ${ }^{13} \mathrm{C}$ nuclei and $\Delta=$ $\Delta_{0}+\Gamma \gamma h(\theta, \phi, t)$. The $\Gamma$ value characterizes the degree of distribution in the magnitude of the magnetic moment. As shown in Fig. 5(b), the observed spectral shape is well reproduced by the simulation with $\Delta_{0}=0.015 \mathrm{MHz}$ and $\Gamma=0.02$, suggesting that the distribution of the local moments in magnitude is around $2 \%$. We note that the sharp central peak, which appears in both the experiments and the simulations, comes from the spin flop because the powder spectrum in the nonflopped case is analytically shown to have a rectangular shape with no anomaly around the origin.

\section{APPENDIX B: SIMULATION of ${ }^{1} \mathrm{H}$ NMR POWDER SPECTRA FOR THE ANTIFERROMAGNETIC STATE}

To examine the possible contribution of the $d p \sigma$ spins to the antiferromagnetic (AF) moment in $\mathrm{Au}(\mathrm{tmdt})_{2}$, we simulated the ${ }^{1} \mathrm{H}$ NMR spectra expected for a polycrystal. According to the molecular-orbital calculations [17,24], the spin population on the ${ }^{1} \mathrm{H}$ sites is negligibly small. Thus, we assume that a local field at a ${ }^{1} \mathrm{H}$ site is mainly generated by the dipole fields of the electron spins of $S=1 / 2$ populated in the $p \pi$ and/or $d p \sigma$ orbitals. In the present magnetic field of $3.7 \mathrm{~T}$, the AF moments should flop to a direction perpendicular to the applied field, and the isotropic hyperfine coupling, if any, has no contribution to the line broadening. We consider local fields at the ${ }^{1} \mathrm{H}$ sites from the $p \pi$ or $d p \sigma$ spins in six neighboring molecules as well as in the on-site molecule. As in our simulation of ${ }^{13} \mathrm{C}$ NMR spectra, we assumed that each microcrystal in our polycrystalline sample is randomly oriented against the field direction. As the magnetic anisotropy of the AF state is not known, we assumed that the flopped moments are directed in an arbitrary direction perpendicular to the external field; namely, we summed the ${ }^{1} \mathrm{H}$ NMR spectra simulated for spins directing randomly in the plane. In addition, we considered the quartet structure of the spectra arising from the nuclear dipolar coupling between nearest-neighbor ${ }^{1} \mathrm{H}$ sites suffering from different local fields in the trimethylene group. Since the distance and direction between the ${ }^{1} \mathrm{H}$ site and the nearest $p \pi$ or $d p \sigma$ spin differ from site to site among the six ${ }^{1} \mathrm{H}$ 's, the local field is also different from site to site. Thus, using the atomic parameters determined by an x-ray diffraction

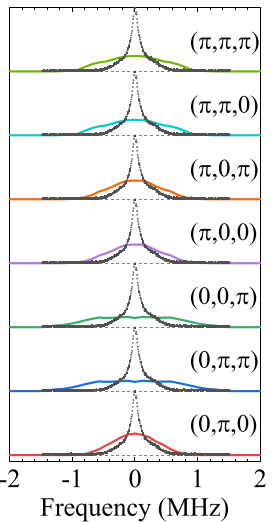

(a) $p \pi$-IAF (b) $p \pi-\mathrm{IF}$

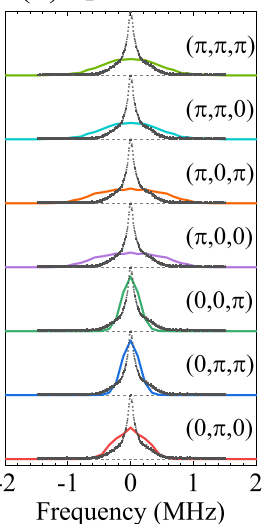

(c) $d p \sigma$

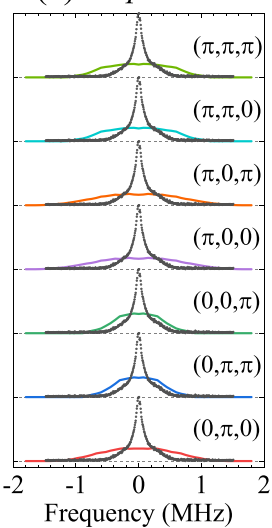

FIG. 7. Simulations of ${ }^{1} \mathrm{H}$ NMR spectra in the antiferromagnetic state. (a, b) The simulated ${ }^{1} \mathrm{H}$ NMR powder spectra of intramolecular antiferromagnetic [IAF (a)] and ferromagnetic [IF (b)] states for the AF moment of $1 \mu_{\mathrm{B}}$ per tmdt with each wave vector $\boldsymbol{q}$. (c) The simulated ${ }^{1} \mathrm{H}$ NMR powder spectra for the AF moment of $1 \mu_{\mathrm{B}}$ per $d p \sigma$ orbital with each wave vector $\boldsymbol{q}$. The experimental ${ }^{1} \mathrm{H}$ NMR spectrum at $2.1 \mathrm{~K}$ (black dots) taken from Ref. [28] is also plotted.

study and the atomic profile of the Mulliken population, we calculated the ${ }^{1} \mathrm{H}$ powder spectra for each of the six ${ }^{1} \mathrm{H}$ sites and summed them up to construct the entire spectra. The simulations were performed for all possible AF configurations of the $p \pi$ spins (14 cases) or $d p \sigma$ spins ( 7 cases) exclusively. The cases for the $p \pi$ spins are divided into two categories; spins on tmdt's in a molecule aligned antiparallel or parallel (7 cases in each), named intramolecular antiferromagnetic (IAF) and intramolecular ferromagnetic (IF) configurations, respectively. The IAF state with the AF wave vector of $\boldsymbol{q}=$ $(\pi, 0,0)$ is suggested to occur by the study of the Hubbard model based on the first-principles band-structure calculations [16-18,24].

Figures 7(a) and 7(b) display the simulated spectra for the IAF and IF states with a moment of $1 \mu_{\mathrm{B}} /$ tmdt, and Fig. 7(c) shows simulations for the $d p \sigma$ spins with a moment of $1 \mu_{\mathrm{B}}$. As seen in the figures, ${ }^{1} \mathrm{H}$ powder spectra are sensitive to $\boldsymbol{q}$, reflecting the form factor of the proton sites. However, it is evident that any case does not reproduce the experimentally observed line shape, even if the magnitude of the moment is varied; namely, the horizontal axis for the simulations is rescaled. This suggests that the observed ${ }^{1} \mathrm{H}$ spectrum is not only contributed to by the $p \pi$ moments but also by the $d p \sigma$ moments. The second moment of the spectra also supports that both $p \pi$ and $d p \sigma$ spins have sizable contributions to the $\mathrm{AF}$ moments as discussed in the main text.

\section{APPENDIX C: ESTIMATION OF INTRAMOLECULAR CHARGE TRANSFER}

It is known that the molecular charge of TTF derivatives such as TMTTF (tetramethyltetrathiafulvalene) and BEDTTTF (Bis(ethylenedithio)tetrathiafulvalene) in solids has a linear relation with the ratio $\gamma=d_{\mathrm{C}=\mathrm{C}} / d_{\mathrm{C}-\mathrm{S}}$ or the difference $\delta=d_{\mathrm{C}-\mathrm{S}}-d_{\mathrm{C}=\mathrm{C}}[37,38]$. Because tmdt has the TTF skeleton, the linearity between the charge and $\gamma$ or $\delta$ is expected to 


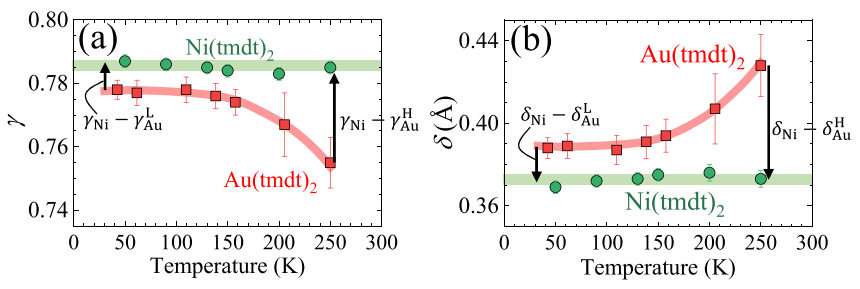

FIG. 8. Estimation of charge transfer from bond length analysis. The ratio of the $\mathrm{C}=\mathrm{C}$ bond length to the $\mathrm{C}-\mathrm{S}$ bond length, $\gamma=$ $d_{\mathrm{C}=\mathrm{C}} / d_{\mathrm{C}-\mathrm{S}}(\mathrm{a})$, and their difference, $\delta=d_{\mathrm{C}-\mathrm{S}}-d_{\mathrm{C}=\mathrm{C}}(\mathrm{b})$. The green lines indicate the levels of $\gamma$ and $\delta$ for $\mathrm{Ni}(\mathrm{tmdt})_{2}$ (averaged over the whole data) corresponding to (tmdt $)^{-1}$. The deviations from the green-line levels are proportional to additional electron occupation in tmdt.

hold for tmdt as well. Given that the valence of tmdt is -1 for $\mathrm{Ni}$ (tmdt $)_{2}$ and -1.5 for $\mathrm{Au}(\mathrm{tmdt})_{2}$ at $250 \mathrm{~K}$, the difference in $\gamma$ or $\delta$ between the two compounds corresponds to the charge of $0.5 e$. Thus, the charge transfer from the $p \pi$ orbital to the $d p \sigma$ orbital, $\Delta Q$, upon cooling from $250 \mathrm{~K}$ to below $T_{\mathrm{N}}$ is estimated through the following relations:

$$
\begin{aligned}
& \Delta Q=\left(1-\frac{\gamma_{\mathrm{Ni}}-\gamma_{\mathrm{Au}}^{\mathrm{L}}}{\gamma_{\mathrm{Ni}}-\gamma_{\mathrm{Au}}^{\mathrm{H}}}\right) 0.5 e, \\
& \Delta Q=\left(1-\frac{\delta_{\mathrm{Ni}}-\delta_{\mathrm{Au}}^{\mathrm{L}}}{\delta_{\mathrm{Ni}}-\delta_{\mathrm{Au}}^{\mathrm{H}}}\right) 0.5 e .
\end{aligned}
$$

Here, $\gamma_{\mathrm{Ni}}$ and $\delta_{\mathrm{Ni}}$ are the temperature-insensitive values for $\mathrm{Ni}(\mathrm{tmdt})_{2}$ indicated by green lines in Fig. 8. $\gamma_{\mathrm{Au}}^{\mathrm{H}}$ and $\delta_{\mathrm{Au}}^{\mathrm{H}}\left(\gamma_{\mathrm{Au}}^{\mathrm{L}}\right.$ and $\left.\delta_{\mathrm{Au}}^{\mathrm{L}}\right)$ are the values of $\mathrm{Au}(\mathrm{tmdt})_{2}$ at $T=250 \mathrm{~K}$ (averaged for $T<T_{\mathrm{N}}$ ) (see Fig. 8). Equations (C1) and (C2) yield $\Delta Q$ of 0.38 and 0.36 , respectively, with $\gamma_{\mathrm{Ni}}=0.785$, $\gamma_{\mathrm{Au}}^{\mathrm{H}}=0.755, \gamma_{\mathrm{Au}}^{\mathrm{L}}=0.778, \delta_{\mathrm{Ni}}=0.373, \delta_{\mathrm{Au}}^{\mathrm{H}}=0.428$, and $\delta_{\mathrm{Au}}^{\mathrm{L}}=0.388$; they are in excellent agreement with each other. Such a large amount of intramolecular charge transfer with temperature variation is extremely unusual but consistent with the anomalous behavior of the NMR shift.
[1] Y. Tokura, S. Seki, and N. Nagaosa, Multiferroics of spin origin, Rep. Prog. Phys. 77, 076501 (2014).

[2] N. A. Spaldin and R. Ramesh, Advances in magnetoelectric multiferroics, Nat. Mater. 18, 203 (2019).

[3] J. Paglione and R. L. Greene, High-temperature superconductivity in iron-based materials, Nat. Phys. 6, 645 (2010).

[4] R. M. Fernandes, A. V. Chubukov, and J. Schmalian, What drives nematic order in iron-based superconductors? Nat. Phys. 10, 97 (2014).

[5] F. Steglich, J. Aarts, C. D. Bredl, W. Lieke, D. Meschede, W. Franz, and H. Schäfer, Superconductivity in the Presence of Strong Pauli Paramagnetism: $\mathrm{CeCu}_{2} \mathrm{Si}_{2}$, Phys. Rev. Lett. 43, 1892 (1979).

[6] G. R. Stewart, Heavy-fermion systems, Rev. Mod. Phys. 56, 755 (1984).

[7] Q. Si and F. Steglich, Heavy fermions and quantum phase transitions, Science 329, 1161 (2010).

[8] S. Kirchner, S. Paschen, Q. Chen, S. Wirth, D. Feng, J. D. Thompson, and Q. Si, Heavy-electron quantum criticality and single-particle spectroscopy, Rev. Mod. Phys. 92, 011002 (2020).

[9] H. Tanaka, Y. Okano, H. Kobayashi, W. Suzuki, and A. Kobayashi, A three-dimensional synthetic metallic crystal composed of single-component molecules, Science 291, 285 (2001).

[10] A. Kobayashi, E. Fujiwara, and H. Kobayashi, Singlecomponent molecular metals with extended-TTF dithiolate ligands, Chem. Rev. 104, 5243 (2004).

[11] R. Kato, H. Kobayashi, and A. Kobayashi, Crystal and electronic structures of conductive anion-radical salts, (2,5$\mathrm{R}_{1}, \mathrm{R}_{2}$-DCNQI) $2 \mathrm{Cu}$ (DCNQI $=N, N^{\prime}$-dicyanoquinonediimine; $\left.\mathrm{R}_{1}, \mathrm{R}_{2}=\mathrm{CH}_{3}, \mathrm{CH}_{3} \mathrm{O}, \mathrm{Cl}, \mathrm{Br}\right)$, J. Am. Chem. Soc. 111, 5224 (1989).

[12] K. Krogmann and H.-D. Hausen, Strukturen mit Pt-Ketten, I. "Violettes" Kaliumtetracyanoplatinat, $\mathrm{K}_{2}\left[\mathrm{Pt}(\mathrm{CN})_{4}\right] \mathrm{X}_{0,3} \cdot 2,5$ $\mathrm{H}_{2} \mathrm{O}(\mathrm{X}=\mathrm{Cl}, \mathrm{Br})$, Z. Anorg. Allg. Chem. 358, 67 (1968).

[13] F. A. Cotton, G. Wilkinson, C. A. Murillo, and M. Bochmann, Advanced Inorganic Chemistry, 6th ed. (Wiley \& Sons, New York, 1999).
[14] M. R. Mian, H. Iguchi, S. Takaishi, H. Murasugi, T. Miyamoto, H. Okamoto, H. Tanaka, S. Kuroda, B. K. Breedlove, and M. Yamashita, Multiple-hydrogen-bond approach to uncommon Pd(III) oxidation state: A Pd-Br chain with high conductivity and thermal stability, J. Am. Chem. Soc. 139, 6562 (2017).

[15] C. Rovira, J. J. Novoa, J.-L. Mozos, P. Ordejón, and E. Canadell, First-principles study of the neutral molecular metal Ni(tmdt $)_{2}$. Phys. Rev. B 65, 081104(R) (2002).

[16] H. Seo, S. Ishibashi, Y. Otsuka, H. Fukuyama, and K. Terakura, Electronic states of single-component molecular conductors $\left[M(\text { tmdt })_{2}\right]$, J. Phys. Soc. Jpn. 82, 054711 (2013).

[17] S. Ishibashi, K. Terakura, and A. Kobayashi, Electronic structures of single component molecular metals based on ab initio calculation, J. Phys. Soc. Jpn. 77, 024702 (2008).

[18] H. Seo, S. Ishibashi, Y. Okano, H. Kobayashi, A. Kobayashi, H. Fukuyama, and K. Terakura, Single-component molecular metals as multiband $\pi-d$ systems, J. Phys. Soc. Jpn. 77, 023714 (2008).

[19] S. Ishibashi and K. Terakura, Electronic structure of $\mathrm{Cu}(\mathrm{tmdt})_{2}$ studied with first-principles calculations, Crystals 2, 1210 (2012).

[20] R. Takagi, K. Miyagawa, M. Yoshimura, H. Gangi, K. Kanoda, B. Zhou, Y. Idobata, and A. Kobayashi, Electronic states and molecular dynamics of single-component molecular conductors $\left[M(\text { tmdt })_{2}\right](M=\mathrm{Ni}, \mathrm{Pt})$ studied by ${ }^{13} \mathrm{C}$ and ${ }^{1} \mathrm{H}$ NMR, Phys. Rev. B 93, 024403 (2016).

[21] B. Zhou, H. Yajima, A. Kobayashi, Y. Okano, H. Tanaka, T. Kumashiro, E. Nishibori, H. Sawa, and H. Kobayashi, Singlecomponent molecular conductor $\left[\mathrm{Cu}(\mathrm{tmdt})_{2}\right]$ containing an antiferromagnetic Heisenberg chain, Inorg. Chem. 49, 6740 (2010).

[22] R. Takagi, K. Miyagawa, K. Kanoda, B. Zhou, A. Kobayashi, and H. Kobayashi, NMR evidence for antiferromagnetic transition in the single-component molecular system $\left[\mathrm{Cu}(\mathrm{tmdt})_{2}\right]$, Phys. Rev. B 85, 184424 (2012).

[23] R. Takagi, T. Hamai, H. Gangi, K. Miyagawa, B. Zhou, A. Kobayashi, and K. Kanoda, Single-component molecular 
material hosting antiferromagnetic and spin-gapped Mott subsystems, Phys. Rev. B 95, 094420 (2017).

[24] S. Ishibashi, H. Tanaka, M. Kohyama, M. Tokumoto, A. Kobayashi, H. Kobayashi, and K. Terakura, Ab Initio electronic structure calculation for single-component molecular conductor $\mathrm{Au}(\mathrm{tmdt})_{2}$ (tmdt $=$ Trimethylenetetrathiafulvalenedithiolate), J. Phys. Soc. Jpn. 74, 843 (2005).

[25] W. Suzuki, E. Fujiwara, A. Kobayashi, Y. Fujishiro, E. Nishibori, M. Takata, M. Sakata, H. Fujiwara, and H. Kobayashi, Highly conducting crystals based on singlecomponent gold complexes with extended-TTF dithiolate ligands, J. Am. Chem. Soc. 125, 1486 (2003).

[26] B. Zhou, M. Shimamura, E. Fujiwara, A. Kobayashi, T. Higashi, E. Nishibori, M. Sakata, H. Cui, K. Takahashi, and H. Kobayashi, Magnetic transitions of single-component molecular metal $\left[\mathrm{Au}(\mathrm{tmdt})_{2}\right]$ and its alloy systems, J. Am. Chem. Soc. 128, 3872 (2006).

[27] H. Tanaka, S. Hara, M. Tokumoto, A. Kobayashi, and H. Kobayashi, Resistance measurements of microcrystals of single-component molecular metals using finely patterned interdigitated electrodes, Chem. Lett. 36, 1006 (2007).

[28] Y. Hara, K. Miyagawa, K. Kanoda, M. Shimamura, B. Zhou, A. Kobayashi, and H. Kobayashi, NMR evidence for antiferromagnetic transition in the single-component molecular conductor, $\left[\mathrm{Au}(\mathrm{tmdt})_{2}\right]$ at $110 \mathrm{~K}$, J. Phys. Soc. Jpn. 77, 053706 (2008).

[29] B. Zhou, A. Kobayashi, Y. Okano, H. B. Cui, D. Graf, J. S. Brooks, T. Nakashima, S. Aoyagi, E. Nishibori, M. Sakata, and H. Kobayashi, Structural anomalies associated with antiferromagnetic transition of single-component molecular metal [Au(tmdt) $)_{2}$ ], Inorg. Chem. 48, 10151 (2009).

[30] L. Binet, J. M. Fabre, C. Montginoul, K. B. Simonsen, and J. Becher, Preparation and chemistry of new unsymmetrically substituted tetrachalcogenofulvalenes bearing $\mathrm{CN}\left(\mathrm{CH}_{2}\right)_{2} \mathrm{X}$ and $\mathrm{HO}\left(\mathrm{CH}_{2}\right)_{2} \mathrm{X}$ groups $(\mathrm{X}=\mathrm{S}$ or $\mathrm{Se})$, J. Chem. Soc., Perkin Trans. 1, 783 (1996).

[31] L. Binet, C. Montginoul, J. M. Fabre, L. Ouahab, S. Golhen, and J. Becher, Preparation of asymmetrical functionalized TTF: Characterization of their charge transfer salts, Synth. Met. 86, 1825 (1997).

[32] K. Sugimoto, H. Ohsumi, S. Aoyagi, E. Nishibori, C. Moriyoshi, Y. Kuroiwa, H. Sawa, and M. Takata, Extremely high resolution single crystal diffractometory for orbital resolution using high energy synchrotron radiation at SPring-8, in SRI 2009, 10th International Conference on Radiation Instrumentation, edited by R. Garrett, I. Gentle, K. Nugent, and S.
Wilkins, AIP Conf. Proc. No. 1234 (AIP, New York, 2010), p. 887.

[33] G. M. Sheldrick, Crystal structure refinement with SHELXL, Acta Crystallogr., Sect. C: Struct. Chem. 71, 3 (2015).

[34] A. Kawamoto, K. Miyagawa, Y. Nakazawa, and K. Kanoda, Electron correlation in the $\kappa$-phase family of BEDT-TTF compounds studied by ${ }^{13} \mathrm{C}$ NMR, where BEDT-TTF is bis(ethylenedithio)tetrathiafulvalene, Phys. Rev. B 52, 15522 (1995).

[35] A. Kawamoto, K. Miyagawa, Y. Nakazawa, and K. Kanoda, ${ }^{13}$ C NMR Study of Layered Organic Superconductors Based on BEDT-TTF Molecules, Phys. Rev. Lett. 74, 3455 (1995).

[36] S. M. De Soto, C. P. Slichter, A. M. Kini, H. H. Wang, U. Geiser, and J. M. Williams, ${ }^{13} \mathrm{C}$ NMR studies of the normal and superconducting states of the organic superconductor $\kappa$ $(\mathrm{ET})_{2} \mathrm{Cu}\left[\mathrm{N}(\mathrm{CN})_{2}\right] \mathrm{Br}$, Phys. Rev. B 52, 10364 (1995).

[37] T. C. Umland, S. Allie, T. Kublmann, and O. Coppens, Relation between geometry and charge transfer in low-dimensional organic salts, J. Phys. Chem. 92, 6456 (1988).

[38] P. Guionneau, C. J. Kepert, G. Bravic, D. Chasseau, M. R. Truter, M. Kurmoo, and P. Day, Determining the charge distribution in BEDT-TTF salts, Synth. Met. 86, 1973 (1997).

[39] T. Takahashi, Y. Maniwa, H. Kawamura, and G. Saito, Determination of SDW characteristics in (TMTSF) ${ }_{2} \mathrm{PF}_{6}$ by ${ }^{1} \mathrm{H}-\mathrm{NMR}$ analysis, J. Phys. Soc. Jpn. 55, 1364 (1986).

[40] K. Miyagawa, A. Kawamoto, K. Uchida, and K. Kanoda, Commensurate magnetic structure in $\kappa$-(BEDT-TTF) $2 X$, Phys. B (Amsterdam, Neth.) 284-288, 1589 (2000).

[41] H. Seo, C. Hotta, and H. Fukuyama, Toward systematic understanding of diversity of electronic properties in low-dimensional molecular solids, Chem. Rev. 104, 5005 (2004).

[42] Y.-Z. Zhang, H. Lee, H.-Q. Lin, C.-Q. Wu, H. O. Jeschke, and R. Valentí, General mechanism for orbital selective phase transitions, Phys. Rev. B 85, 035123 (2012).

[43] A. Himeda and M. Ogata, Coexistence of $d_{x^{2}-y^{2}}$ superconductivity and antiferromagnetism in the two-dimensional $t-J$ model and numerical estimation of Gutzwiller factors, Phys. Rev. B 60, R9935 (1999).

[44] T. Miyazaki and K. Terakura, First-principles theoretical study of metallic states of DCNQI-(Cu, Ag, Li) systems, Phys. Rev. B 54, 10452 (1996).

[45] R. Kato, Conductive copper salts of 2,5-disubstituted $N, N^{\prime}$ dicyanobenzoquinonediimines (DCNQIs): Structural and physical properties, Bull. Chem. Soc. Jpn. 73, 515 (2000).

[46] K. Kanoda, Metal-insulator transition in $\kappa$-(ET) $)_{2} \mathrm{X}$ and $(\mathrm{DCNQI})_{2} \mathrm{M}$ : Two contrasting manifestation of electron correlation, J. Phys. Soc. Jpn. 75, 051007 (2006). 